\title{
Effect of body mass index on pulmonary function in children
}

\author{
G Liyanage', B D Jayamanne'2, M Aaqiff ${ }^{3}$, D Sriwardhana ${ }^{3}$
}

(Index words: pulmonary function, spirometry, obesity, overweight, Sri Lanka)

\begin{abstract}
Introduction The relationship between obesity or overweight and pulmonary functions has not been investigated in Sri Lankan children previously. Therefore, we aimed to study the effects of obesity / overweight on dynamic lung functions in healthy school children in Sri Lanka.
\end{abstract}

Methods This cross sectional study was carried out among healthy school children. They were categorised into 'obese/over weight' group (BMI $>85^{\text {th }}$ percentile) and 'normal weight' group (BMI $5^{\text {th }}-85^{\text {th }}$ percentile). Forced vital capacity (FVC), forced expiratory volume in one second (FEV1) and forced expiratory flow at 25-75\% (FEF 25-75\%) were used as measures of ventilatory function.

Results Of 275 children 55 (20\%) were 'obese/ overweight'. BMI has no significant relationship to any of the pulmonary function parameters. However, in contrast to what is expected, mean values of $\mathrm{FVC}, \mathrm{FEV}$, and FEF $25-75 \%$ were lower in 'normal weight' group (Table 2).

Conclusions No significant difference was detected in spirometry parameters between obese/overweight and normal weight school children in our study.

Ceylon Medical Journal 2016; 61: 163-166

http://doi.org/10.4038/cmj.v61i4.8382

\section{Introduction}

Prevalence of obesity and overweight is increasing in adults and in children in both developed and developing countries $[1,2]$. Obesity is a risk factor for many conditions including respiratory disorders such as asthma, increased airway resistance and atopy $[3,4]$. Therefore, increasing numbers of obese children with respiratory disorders are referred for lung function testing. Pulmonary function abnormalities are recognised complications of obesity in adults [5]. However, available data on lung functions of children with overweight and obesity are inconsistent [6-8]. Further, there is limited information on effect of
BMI on lung function in children in Sri Lanka. Therefore, this study was designed to determine the effects of obesity and overweight on dynamic lung functions measured with spirometry.

\section{Methods}

Children between 9-15 years were recruited for this cross-sectional survey which was conducted in Colombo District in Sri Lanka. Sample size was calculated based on observed variance of dynamic lung volumes from a previous study and prevalence of overweight/obesity (14\%) among healthy school children in the Colombo metropolitan area [7,9]. Three schools with both girls and boys and primary and secondary education were randomly chosen. In each selected school, one class from each grade was randomly selected to recruit children between 9-15 years. All the students in each class were invited for the study. The principal and the teachers were given general information about spirometry and a detailed explanation of the procedure. Informed written consent was obtained from the parents. A pretested questionnaire based on International Study of Asthma and Allergies in Childhood (ISAAC) questionnaire was distributed among the parent/guardian of the selected children [10]. It recorded information on demography, past and current medical/ surgical problems and exposure to environmental triggers. Subsequently all the children who returned the completed questionnaire with the signed consent form underwent a detailed clinical assessment by the principal investigator.

Children who had upper or lower respiratory infections, chronic respiratory disorders such as asthma or interstitial lung disease, congenital abnormalities of cardio-respiratory system, chest deformities and neuromuscular diseases were excluded.

Subsequently all children who fulfilled the inclusive criteria, underwent spirometry. The weight $(\mathrm{kg})$ and standing height $(\mathrm{cm})$ were measured with a calibrated weighing scale and a stadiometer. Spirometry was carried out by two trained technicians. We measured

${ }^{1}$ Department of Paediatrics, Faculty of Medical Sciences, ${ }^{2}$ University of Sri Jayewardenepura, National Dengue Control Unit, Ministry of Health, ${ }^{3}$ University Paediatric Unit, Colombo South Teaching Hospital, Sri Lanka.

Correspondence: GL, e-mail: <guwani@sjp.ac.Ik>. Received 12 May 2015 and revised version accepted 18 April 2016. 
pulmonary function parameters using an Alpha Touch Vitalograph Spirometer in accordance with the American Thoracic Society and European Respiratory Society Guidelines. The spirometer was calibrated each day using a three litre syringe. All children performed forced expiratory manoeuvres to obtain optimal effort (duration of exhalation at least $3 \mathrm{~s}$ for children $<11$ years old and $6 \mathrm{~s}$ for children $>11$ years old). The best of at least three technically accep-table values for FEV1, FVC and flow volume curves were selected. These children with acceptable spirometry values were grouped according to BMI. 'Obese/overweight'group included children with BMI for the age and sex more than the 85th percentile according to CDC (Centre for Diseases Control and Prevention) reference charts. Overweight was defined as BMI between 85 th and 95th percentile and obesity as BMI $>95$ th percentile. Children between 5 th and 85 th centile were considered 'normal weight'. BMI with $<5$ th percentile was defined as 'underweight'.

Our study was approved by the Ethics Committee of the Faculty of Medical Sciences, University of Sri Jayewardenepura Sri Lanka. Pearson's chi-square test was used to evaluate potential association with categorical variables and student's t-test was used to assess mean differences with ratio-scale variables. SPSS for Windows statistical software was used for the analysis. The level of significance was set at $5 \%$ in all comparisons.

\section{Results}

Four hundred and five completed questionnaires were returned. Of them 93 were excluded due to incomplete data. Two hundred and seventy five healthy children had acceptable and reproducible spirometry curves. Finally results of 275 children were analysed. Of them, $55(20 \%)$ were in the 'obese/overweight' category. In $198(72 \%)$ BMI was in the normal range (5th to 85th percentile). A subgroup of these normal children was randomly selected (a random sub sample) to compare with the obese/overweight group. Characteristics of this random sub sample of normal weight children were similar to the characteristics of the whole group of normal weight children and no significant differences were noted with regard to their age, gender, height or weight. Then the results of these two groups were compared. The demographic data are given in table 1.

In our study BMI has no significant relationship to any of the pulmonary function parameters. However, in contrast to what is expected, mean values of FVC, $\mathrm{FEV}_{1}$ and FEF $25-75 \%$ were lower in 'normal weight' group (Table 2).

Table 1. Characteristics of the 'obese/overweight' and 'normal weight' groups

\begin{tabular}{|c|c|c|c|}
\hline & $\begin{array}{c}\text { Obese/overweight } \\
n=55\end{array}$ & $\begin{array}{c}\text { Normal weight } \\
n=64\end{array}$ & $p$ value \\
\hline Mean age in years (SD) & $11.76(1.55)$ & $11.94(1.50)$ & 0.62 \\
\hline Mean BMI (SD) & $25.20(4.36)$ & $16.20(1.70)$ & 0.00 \\
\hline Mean height in centimeters (SD) & $142.9(16.60)$ & $147.56(11.21)$ & 0.07 \\
\hline Male: female ratio & $31: 24$ & $31: 33$ & 0.39 \\
\hline Exposure to cigarette or other smoke & 11 & 13 & 0.58 \\
\hline Family history of asthma & 13 & 19 & 0.30 \\
\hline
\end{tabular}

Table 2. Comparison of dynamic lung volumes among 'obese/overweight' and 'normal weight' groups

\begin{tabular}{|c|c|c|c|c|c|c|c|}
\hline & \multicolumn{2}{|c|}{$\begin{array}{l}\text { Obese/overweight } \\
\left(B M I>85^{\text {th }} \text { percentile }\right)\end{array}$} & \multicolumn{2}{|c|}{$\begin{array}{l}\text { Normal weight } \\
\text { (BMI } 5^{\text {th }}-85^{\text {th }} \text { percentile) }\end{array}$} & \multirow[t]{2}{*}{$M f$} & \multirow[t]{2}{*}{$95 \% C I$} & \multirow[t]{2}{*}{$p$ value } \\
\hline & Mean & $S D$ & Mean & $S D$ & & & \\
\hline $\mathrm{FVC}$ & 2.36 & 0.07 & 2.19 & 0.06 & 0.17 & $(-0.02,0.37)$ & 0.90 \\
\hline FEV1 & 2.05 & 0.06 & 1.93 & 0.05 & 0.11 & $(-0.05,0.28)$ & 0.17 \\
\hline FEV1/FVC & 83.89 & 2.26 & 87.85 & 0.61 & -3.95 & $(-8.32,0.40)$ & 0.07 \\
\hline FEF $25-75 \%$ & 2.49 & 0.09 & 2.37 & 0.06 & 0.12 & $(-0.10,0.34)$ & 0.29 \\
\hline
\end{tabular}

(FEV1/ FVC: ratio of FEV1 to FVC) 


\section{Discussion}

The reasons for the link between obesity and res-piratory disorders are not completely understood. Several possible mechanisms related to obesity have been investigated which may be causing respiratory problems especially asthmatic symptoms in obese individuals [11-13]. It has been correlated to mechanical effect of adipose tissue on the lung, airway inflammation and bronchial hyper responsiveness. The exact causative mechanisms has not been established. Previous studies have not firmly established the relationship between obesity and spirometry parameters. Some of these studies have shown a significant reduction in spirometry parameters with obesity while others have not shown any effect [6-8]. Our study did not show any significant effect of obesity or overweight on dynamic lung volumes measured by spirometer.

Lazarus et al has examined the effect of total body fat as a percentage of weight on FVC and FEV1 [6]. Total body fat was estimated by measuring skin fold thickness. He showed that height-adjusted FVC and FEV1 values increased significantly with increment in weight for age and sex. Since weight measures both lean and fat mass, comparison after adjusting FVC and FEV1 values for both height and weight showed a significant decrease of ventilator functions with increasing skin fold thickness. Turan and co-workers have shown in a comparative study on obese and non-obese that although there was no statistically significant difference in $\mathrm{FEV}_{1}, \mathrm{FVC}$, or FEV1/ $\mathrm{FVC}$, a significant reduction in peak expiratory flow rate (PEFR) and FEF 25-75\% was found in overweight and obese individuals [7].

This discrepancy observed between studies investigating relationship between spirometry parameters and obesity/overweight could be explained in many ways. Methodological differences such as dissimilar criteria in measuring the fat mass or sample size may have played a role. Further, distribution of body fat in different populations, degree of obesity and duration of obesity in the samples recruited may have an effect on pulmonary function. This variability in results may also be due to positive effect of growth masking the negative effects of obesity on lung function.

In general, BMI is considered a measure of obesity and it cannot distinguish fat and lean body mass [14]. It is an index of nutritional status and its relationship with body composition is controversial. When compared with other measurements, accuracy and precision of skin fold thickness measurements is poor in obese children [14]. However, it can be used to predict body composition. Waist circumference provides a simple measure of central fatness and it may have a direct effect on the chest wall properties. In addition, there are newer sophisticated techniques of assessing fat mass in children. Bioelectric impendence analysis (BIA) and Dual energy X-ray absorptiometry (DXA) have the potential to improve accuracy and predicts the body composition. Therefore, selecting a method which measures body fat composition accurately would have given different results to what we have found in this study.

Some studies have investigated the relationship between obesity and static lung volumes (total lung capacity, functional residual capacity, residual volume), and ventilation-perfusion relationships in children. In that context, $\mathrm{Li}$ et al has shown that increased body fat measured by DXA scan has a negative correlation with functional residual capacity [15].

There are few limitations in our study. A bigger sample size would have given more concrete evidence. Further, although BMI is the most widely used acceptable method of obesity, it does not differentiate the lean mass and fat mass in an individual. Therefore, a method which assesses the body composition would have given more accurate results. In our study we have only investigated the dynamic lung volumes since we did not have facilities to examine static lung volumes and ventilation-perfusion relationship in children. However, recruiting healthy children following a strict screening procedure carried out by the principal investigator is a strength in our study.

In conclusions, our study did not show a significant effect of obesity/overweight on dynamic lung volumes among healthy school children in Colombo District, Sri Lanka.

\section{Acknowledgements}

The authors like the Technical Officer, Asitha Weerasinghe of the Department of Paediatrics, Faculty of Medical Sciences, University of Sri Jayewardenepura.

\section{Conflicts of interest}

There are no conflicts of interest.

\section{References}

1. Katulanda MA, Jayawardena MH, Sheriff GR, Constantine DR. Prevalence of overweight and obesity in Sri Lankan adults. Obes Rev 2010; 11: 751-6.

2. WHO. Obesity: preventing and managing the global epidemic: report of a WHO consultation. WHO technical report series; 894. Geneva: The Office of Publication, World Health Organization, 1999.

3. Zammit C, Liddicoat H, Moonsie I, Makker H. Obesity and respiratory diseases. Int J Gen Med 2010; 3: 335-43.

4. Choudhary AK, Donnelly LF, Racadio, Strife JL. Diseases Associated with Childhood Obesity. Am J Roentgenol 2007; 188: $1118-30$.

5. Jones RL, Nzekwu MM. The effect of body mass index on lung volumes. Chest 2006; 130: 827-33. 
6. Lazarus R, Colditz G, Berkey CS, Speizer FE. Effects of body fat on ventilatory function in children and adolescents: Cross-sectional findings from a random population sample of school children. Pediatr Pulmonol 1997; 24: 187-94.

7. Torun E, Cakir E, Ozguç F, Ozgen IT. The Effect of Obesity Degree on Childhood Pulmonary FunctionTests. Balkan Med $J$ 2014; 31: 235-8.

8. Paralikar SJ, Kathrotia RG, Pathak NR, Jani MB Assessment of pulmonary functions in obese adolescent boys. Lung India 2012; 29: 236-40.

9. Wickramasinghe VP, Lamabadusuriya SP, Cleghorn GJ, Davies PSW. Validity of currently used cutoff values of body mass index as a measure of obesity in Sri Lankan children. Ceylon Med J 2009; 54: 114-9.

10. International Study on Asthma \& Allergies in Childhood. ISAAC Tools \& Manuals. http://isaac.auckland.ac.nz/ resources/tools.php
11. Fantuzzi G. Adipose tissue, adipokines, and inflammation. J Allergy Clin Immunol 2005; 115: 911-9.

12. Santamaria F, Montella S, De Stefano S, et al. Asthma, atopy, and airway inflammation in obese children. $J$ Allergy Clin Immunol 2007; 120: 965-7.

13. McLachlan CR, Poulton R, Car G, et al. Adiposity, asthma, and airway inflammation. J Allergy Clin Immunol 2007; 119: 634-9.

14. Wells JCK, Fewtrell MS. Measuring body composition. Arch Dis Child 2006; 91: 612-7.

15. Li AM, Chan D, Wong E, Yin Y, Nelson EA, Fok TF. The effects of obesity on pulmonary function. Arch Dis Child 2003; 88: 361-3. 